\title{
Upregulation and hypomethylation of IncRNA AFAP1-AS1 predicts a poor prognosis and promotes the migration and invasion of cervical cancer
}

\author{
HAO BO ${ }^{1-3}$, LIQING FAN $^{3}$, ZHAOJIAN GONG $^{4}$, ZHIZHONG LIU $^{5}$, LEI SHI $^{2}$, CAN GUO $^{2}$, XIAYU LI ${ }^{2}$, \\ QIANJIN LIAO ${ }^{2,5}$, WENLING ZHANG ${ }^{2}$, MING ZHOU $^{1,2}$, BO XIANG ${ }^{1,2}$, XIAOLING LI ${ }^{1,2}$, \\ GUIYUAN LI ${ }^{1,2}$, WEI XIONG ${ }^{1,2}$, ZHAOYANG ZENG ${ }^{1,2}, \mathrm{KE} \mathrm{CAO}^{6}$, \\ SHANSHAN ZHANG ${ }^{1,2}$ and FANG XIONG ${ }^{1,2}$
}

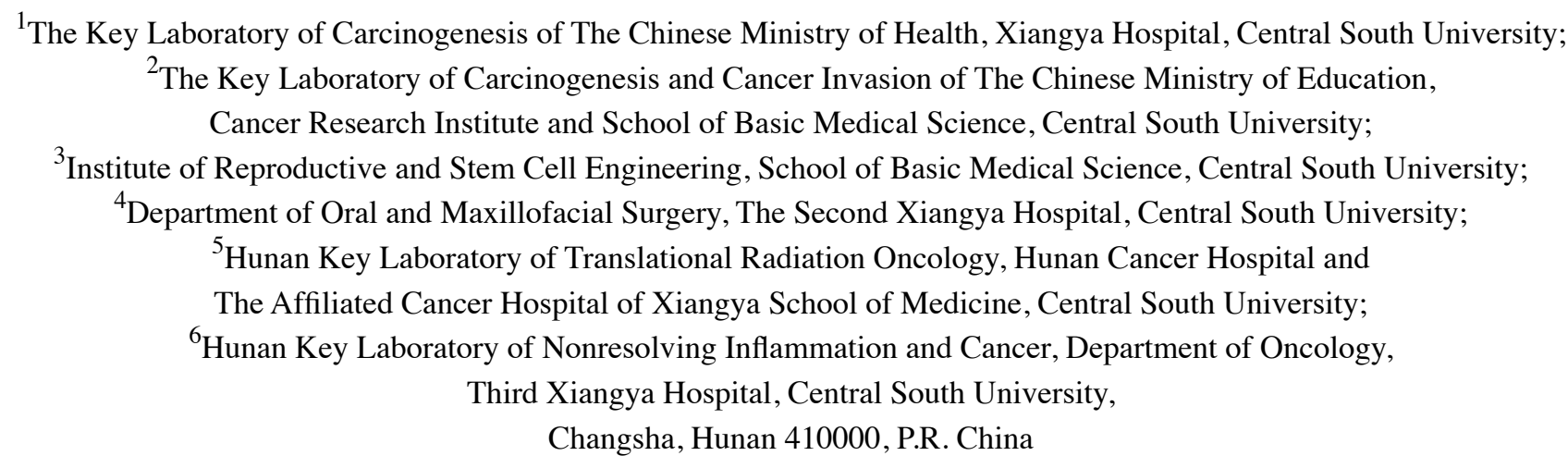

Received June 11, 2018; Accepted January 16, 2019

DOI: $10.3892 /$ or.2019.7027

\begin{abstract}
Although lncRNA AFAP1 antisense RNA1 (AFAP1-AS1) is considered an oncogenic lncRNA, little is known about the role of AFAP1-AS1 in cervical cancer. In the present study, we found that AFAP1-AS1 was elevated and hypomethylated in cervical cancer and was associated with a poor prognosis of patients with cervical cancer by analyzing the Cancer RNA-Seq Nexus (CRN), MethCH and UCSC XENA databases. Subsequently, we knocked AFAP1-AS1 expression down using siRNAs in cervical cancer cells. Wound healing experiments and matrigel invasion experiments revealed that
\end{abstract}

Correspondence to: Dr Fang Xiong or Dr Shanshan Zhang, The Key Laboratory of Carcinogenesis of The Chinese Ministry of Health, Xiangya Hospital, Central South University, 87 Xiangya Road, Changsha, Hunan 410008, P.R. China

E-mail: xiongf@csu.edu.cn

E-mail: zhangshanshan@csu.edu.cn

Abbreviations: AFAP1-AS1, AFAP1 antisense RNA1; ANOVA, analysis of variance; ATCC, American Type Culture Collection; CRN, Cancer RNA-Seq Nexus; EMT, epithelial-mesenchymal transition; GEO, Gene Expression Omnibus; lncRNAs, long non-coding RNAs; OS, overall survival; PI, propidium iodide; SDS-PAGE, sodium dodecyl sulfate-polyacrylamide gel electrophoresis; SE, standard error; siRNAs, short interfering RNAs; SRA, Sequence Read Archive; TCGA, The Cancer Genome Atlas

Key words: long non-coding RNAs, AFAP1 antisense RNA1, cervical cancer, prognosis, migration and invasion the downregulation of AFAP1-AS1 suppressed the migration and invasion of cervical cancer cells. Furthermore, western blot analysis demonstrated that the antitumor effects induced by the silencing of AFAP1-AS1 were mainly mediated through the regulation of the Rho/Rac signaling pathway and epithelial-mesenchymal transition (EMT)-related genes. Taken together, the findings of the present study indicate that the expression level of AFAP1-AS1 may be involved in the development of cervical cancer. Thus, AFAP1-AS1 may be a novel prognostic biomarker and a potential therapeutic target for patients with cervical cancer.

\section{Introduction}

Cervical cancer, one of the most common malignant tumors affecting the female population, brings a serious problem to affect public women's health (1). The morbidity and mortality associated with cervical cancer has significantly decreased over the past three decades. However, cervical cancer remains the third most commonly diagnosed type of cancer among women worldwide and some patients with this disease have a poor prognosis (2-4). Therefore, novel and effective therapeutic strategies for cervical cancer are urgently required. Accumulating evidence has indicated that the development of cervical cancer is a multi-step process, including the abnormal expression of oncogenes and tumor suppressor genes (5-7). Despite advances in cervical cancer, the precise molecular mechanisms of carcinogenesis and progression underlying cervical cancer are only partly understood (5). Therefore, the identification of the specific molecular biomarkers and 
detailed underlying mechanisms, which may contribute to the development of novel diagnostic and treatment strategies for patients with cervical cancer is critical.

Long non-coding RNAs (lncRNAs) are a distinct subclass of RNA transcripts that more than 200 nucleotides in length (8). An increasing number of individual lncRNAs have been determined to exert a multitude of effects within cells, such as modulating gene expression at the epigenetic, transcriptional, post-transcriptional and translational levels under both physiological and pathological conditions (9-13). The aberrant expression of lncRNAs has been linked to tumor initiation and progression (14-18).

The findings from our previous studies (19-24), as well as other studies (25-27) have reported that lncRNA AFAP1-AS1 is upregulated in several tumors and functions as an oncogene. However, the expression level, epigenetic state and function of AFAP1-AS1 in cervical cancer remain unclear. Thus, in the present study, we firstly analyzed the related databases and found that AFAP1-AS1 expression was increased and that it was hypomethylated in cervical cancer. Furthermore, to evaluate the potential biological functions of AFAP1-AS1, we performed loss-of-function experiments and functional analyses. The findings of the present study may provide novel insight concerning the specific role of AFAP1-AS1 in the development of cervical cancer.

\section{Materials and methods}

Gene expression data and DNA methylation. Cancer RNA-Seq Nexus (CRN) is a database of phenotype-specific transcriptome profiling in cancer cells. Using this database, we systematically obtained RNA-seq datasets concerning cervical cancer tissues from The Cancer Genome Atlas (TCGA), Sequence Read Archive (SRA) and NCBI Gene Expression Omnibus (GEO) (28). The expression data of AFAP1-AS1 in cervical cancer was downloaded from this database. The DNA methylation data of cervical cancer was downloaded from the MethHC database (http://methhc.mbc.nctu.edu.tw/php/index. php), data which came from TCGA (29). The data used to draw the survival curve were all from the UCSC XENA database (http://xena.ucsc.edu/).

Cell lines and siRNAs. The HeLa cell line (ATCC no. CCL-2) is the most widely usedcervical cancer cell line. Due to its strong ability to proliferate, invade and migrate, we selected it for further research. The HeLa cells were purchased from the American Type Culture Collection (ATCC; Manassas, VA, USA) and cultured in RPMI-1640 medium supplemented with $10 \%$ fetal bovine serum (FBS; Thermo Fisher Scientific, Inc., Waltham, MA, USA), penicillin and streptomycin (both from Sigma-Aldrich; Merck KGaA, Darmstadt, Germany) in a humidified incubator with $5 \% \mathrm{CO}_{2}$ at $37^{\circ} \mathrm{C}$. For gene knockdown, the cells were seeded and cultured overnight. Subsequently, $20 \mathrm{nM}$ AFAP1-AS1 siRNA or scramble control (NC) siRNA (Guangzhou RiboBio Co., Ltd., Guangzhou, China) were transfected into the cervical cancer cells using Lipofectamine 3000 (Thermo Fisher Scientific, Inc.) according to the manufacturer's instructions. The two siRNAs used in this manuscript had no off-target effect and exhibited the optimal knockdown efficiency in previous studies by us, as well as other groups $(19,30)$. The cycling conditions of PCR were as follows: First, $10 \mathrm{~min}$ at $95^{\circ} \mathrm{C}$. Denaturation at $95^{\circ} \mathrm{C}$ for $15 \mathrm{sec}$, annealing at $60^{\circ} \mathrm{C}$ for $30 \mathrm{sec}$, and elongation at $70^{\circ} \mathrm{C}$ for $10 \mathrm{sec}$. After 40 cycles, the PCR tubes were incubated $5 \mathrm{~min}$ at $70^{\circ} \mathrm{C}$. The sequences were as follows: AFAP1-AS1 siRNA1, 5'-GGG CTTCAATTTACAAGCATT-3' and AFAP1-AS1 siRNA2, 5'-CCTATCTGGTCAACACGTATT-3'. The abovementioned nucleotide sequences were synthesized by Guangzhou RiboBio Co., Ltd.

Scratch test. The cervical cancer cells were seeded and cultured in 6-well culture plates following transfection for $24 \mathrm{~h}$. When the cells grew to $90 \%$ confluence, a $10 \mu \mathrm{l}$ tip was used to create a scratch. Images were captured (magnification, x20) at different time points $(0,24$ and $48 \mathrm{~h})$ using a microscope (The Cell Culture Laboratory Solution CKX53; Olympus, Tokyo, Japan). The ocular ruler was performed to measure the gap width in each group at the identified time point. All the wounds in the experimental group had the same width at the $0 \mathrm{~h}$ (31-33).

Transwell invasion assay. The invasive capacity was detected by Transwell invasion assays (34-36). The HeLa cells $\left(1 \times 10^{5}\right)$ were transfected with AFAP1-AS1 siRNA or NC siRNA and were plated on the top chamber of a 24-well Transwell insert (8- $\mu \mathrm{m}$ pore size; BD Biosciences, San Jose, CA, USA). Matrigel (20 $\mu \mathrm{l})(2 \mathrm{mg} / \mathrm{ml}$; BD Biosciences) was placed on the top well. The bottom well contained $800 \mu \mathrm{l}$ completed RPMI-1640 medium (containing with $15 \%$ FBS). Following $48 \mathrm{~h}$ of incubation at $37^{\circ} \mathrm{C}$, the cells on the upper surface were removed using a cotton swab, while the cells that had invaded through the matrigel were fixed in $4 \%$ paraformaldehyde for $20 \mathrm{~min}$ and stained with $0.1 \%$ crystal violet (Merck KGaA, Darmstadt, Germany) for $10 \mathrm{~min}$ at room temperature. Invasive tumor cells were observed and calculated under a light microscope (The Cell Culture Laboratory Solution CKX53; Olympus) and 5 randomly low power (x20) fields were selected for each test.

MTT cell viability assay. MTT assay was used to assess cell viability (37-40). The transfected cells were seeded into 96-well plates at a density of $5 \times 10^{3}$ cells/well. Subsequently, $100 \mu 1$ complete RPMI-1640 medium were added per well and cultured for $0,1,2,3,4$ and 5 days. MTT reagent (20 $\mu 1$ of $1 \mathrm{mg} / \mathrm{ml}$; Sigma-Aldrich; Merck KGaA) was then added to each well followed by incubation for $4 \mathrm{~h}$. The absorbance was detected at $450 \mathrm{~nm}$ using a spectrophotometer (Beckman Coulter, Inc., Brea, CA, USA).

RNA isolation and RT-qPCR. The HeLa cells were harvested after being transfected with the siRNAs for $36 \mathrm{~h}$ and total RNA was extracted using TRIzol reagent (Invitrogen/Thermo Fisher Scientific, Inc.) according to the manufacturer's instructions. The RNA was reversed into cDNA using the $5 \mathrm{X}$ All-In-One RT Master Mix Reagent kit (Abm Canada Inc., Milton, ON, Canada) according to the kit protocol. cDNA was subjected to RT-qPCR using the SYBR Premix Ex Taq II kit (Takara Bio, Inc., Shiga, Japan). Reactions were carried out on the CFX96 Real-Time PCR Detection System (185-5196; Bio-Rad Laboratories, Inc., Hercules, CA, USA). Fold changes of lncRNA were calculated using the $2^{-\Delta \Delta \mathrm{Cq}}$ method, with $\beta$-actin 
as an internal control (41). The sequences of the primers were as follows: AFAP1-AS1 forward, 5'-AATGGTGGTAGGAGG GAGGA-3' and reverse, 5'-CACACAGGGGAATGAAGA GG-3'; $\beta$-actin forward, 5'-TCACCAACTGGGACGACA TG-3' and reverse, 5'-GTCACCGGAGTCCATCACGAT-3'.

Cell cycle detection. Cell cycle distribution was detected by flow cytometry. When the cells were transfected with the siRNAs for $48 \mathrm{~h}$, the cells were collected and fixed in $75 \%$ ethanol at $-20^{\circ} \mathrm{C}$ overnight. The fixed cells were then washed thrice with phosphate-buffered saline (PBS) before being incubated at room temperature with RNase A for $20 \mathrm{~min}$. These cells were stained using a propidium iodide (PI) staining kit (BD Biosciences) and incubated in the dark for $30 \mathrm{~min}$ at $4^{\circ} \mathrm{C}$. A Beckman flow cytometer (Beckman Coulter, Inc.) was used for cell cycle analysis. Three independent experiments were conducted.

Western blot analysis. Total protein in the HeLa cells was extracted using RIPA extraction reagent (Beyotime Institute of Biotechnology, Shanghai, China) supplemented with a protease inhibitor cocktail (Roche Diagnostics, Basel, Switzerland). Protein from the HeLa cells was quantified by the BCA method. To separate the protein, 30-50 $\mu \mathrm{g}$ protein per lane was loaded and electrophoresed in $10 \%$ sodium dodecyl sulfate-polyacrylamide gel electrophoresis (SDS-PAGE). Following electrophoresis, the protein bands were transferred onto PVDF membranes. The membranes were blocked with $5 \%$ skim milk for $1 \mathrm{~h}$ at room temperature and then incubated with primary anti-Rho GDP-dissociation inhibitor 1 (RHOGDI, 1:1,000; cat. no. 14282-1-AP), anti-Rac Family Small GTPase 2 (RAC2, 1:1,000; cat. no. 10735-1-AP), anti-RAB1B (1:800; cat. no. 17824-1-AP), anti-RAB11A (1:800; cat. no. 20229-1-AP), anti-profilin 1 (PFN1, 1:1,000; cat. no. 11680-1-AP) and anti-LIM and SH3 protein 1 (LASP1, 1:1,000; cat. no. 10515-1-AP) antibodies (ProteinTech, Inc., Rosemont, IL, USA) or E-cadherin (1:1,000; cat. no. 3195), Zonula occludens-1 (ZO-1, 1:1,000; cat. no. 8193), Vimentin $(1: 1,000$; cat. no. 5741), $\beta$-catenin antibodies $(1: 1,000$; cat. no. 8480) (Cell Signaling Technology, Inc., Danvers, MA, USA) overnight at $4^{\circ} \mathrm{C}$. The following day, the membranes were incubated with a horseradish peroxidase-conjugated secondary antibody (goat anti-mouse IgG-HRP; cat. no. SC-2005; and goat anti-rabbit IgG-HRP, cat. no. SC-2005; 1:2,000; Santa Cruz Biotechnology, Inc., Santa Cruz, CA, USA) for $30 \mathrm{~min}$ at $37^{\circ} \mathrm{C}$. Immunoreactive bands were visualized using an ECL detection reagent (Amersham; GE Healthcare Life Sciences, Chalfont, UK). $\beta$-actin (1:1,000; cat. no. 4970; Cell Signaling Technology, Inc.) expression was used as a housekeeping gene control.

Statistical analysis. All the experimental data are represented as the means \pm standard error (SE) and processed using SPSS statistical software, version 19.0 (SPSS, Inc., Chicago, IL, USA). Overall survival (OS) was analyzed using the Kaplan-Meier method and the results of the analysis were considered significant if log-rank test yielded a value of $P<0.05$. Significance between 2 groups was evaluated by a Student's t-test. Pearson's correlation analysis was used to assess the correlation between AFAP1-AS1 expression and its methylation. For the comparison of multiple groups, analysis of variance (ANOVA) with Dunnett's post hoc t-test was performed. A P-value $<0.05$ was considered to indicate a statistically significant difference.

\section{Results}

Upregulated AFAP1-AS1 expression is associated with a poor prognosis of patients with cervical cancer. Firstly, the expression level of AFAP1-AS1 in cervical cancer samples was analyzed using TCGA data from the CRN database. The data suggested that AFAP1-AS1 was more abundant in the cervical cancer tissues compared to the normal tissues $(\mathrm{P}<0.001$; Fig. 1A). Moreover, a high AFAP1-AS1 expression was positively associated with the TNM stage $(\mathrm{P}<0.05$; Fig. $1 \mathrm{~B})$. To validate whether the expression levels of AFAP1-AS1 were associated with the OS of patients with cervical cancer, we analyzed the patient outcome data from the UCSC XENA database. This analysis revealed that compared to patients with a low expression of AFAP1-AS1, patients with a high AFAP1-AS1 expression had a shorter survival time $(\mathrm{P}<0.05$; Fig. 1C).

Hypomethylation of AFAPI-AS1 is associated with a poor prognosis of patients with cervical cancer. DNA methylation is an important part of epigenetic inheritance and is involved in the transcriptional expression of lncRNAs $(42,43)$. In the present study, to investigate whether the expression of AFAP1-AS1 is influenced by DNA methylation, we downloaded the methylation data of AFAP1-AS1 in cervical cancer from the human pan-cancer method database, MethCH. We found that the average methylation level of the AFAP1-AS1 promoter region was lower in the cervical cancer samples when compared with the non-tumor cervical cancer samples ( $\mathrm{P}<0.01$; Fig. $2 \mathrm{~A})$. In addition, the results of Pearson's correlation analysis revealed that the expression level of AFAP1-AS1 negatively correlated with its methylation level $(r=-0.33, \mathrm{P}<0.001$; Fig. $2 \mathrm{~B})$. The survival analysis was constructed using the UCSC XENA database, and we identified that the hypomethylation of AFAP1-AS1 was associated with a poor overall survival of patients with cervical cancer $(\mathrm{P}=0.05$; Fig. $2 \mathrm{C})$. These data suggested that the high expression of AFAP1-AS1 may be related to the hypomethylation of its promoter region. In addition, the hypomethylation of the AFAP1-AS1 promoter region and the high expression levels of AFAP1-AS1 may act as independent prognostic indicators of patients with cervical cancer.

Tumor cell migration and invasion are suppressed following the knockdown of AFAP1-AS1. To elucidate the role of AFAP1-AS1 in cervical cancer, the AFAP1-AS1 expression was downregulated by transfection of the cervical cancer cell line, HeLa, with two validated siRNAs targeting AFAP1-AS1 (siRNA1 and siRNA2). We examined the knockdown efficiency of these two siRNAs by RT-qPCR which yielded satisfactory results. AFAP1-AS1 expression was knocked down by at least $65 \%$ in the HeLa cells (both siRNAs, $\mathrm{P}<0.01$; Fig. 3A). The functional experiments were performed to examine the phenotypic alterations induced by the silencing of AFAP1-AS1 in the cervical cancer cells. The 


\section{A}

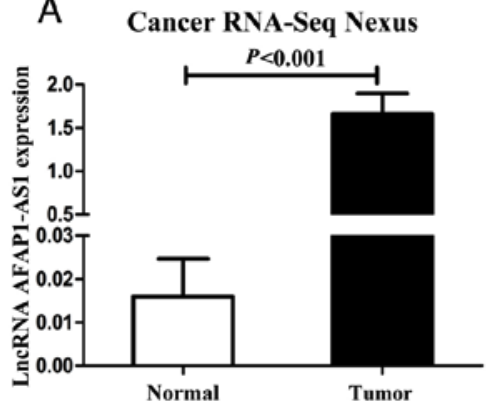

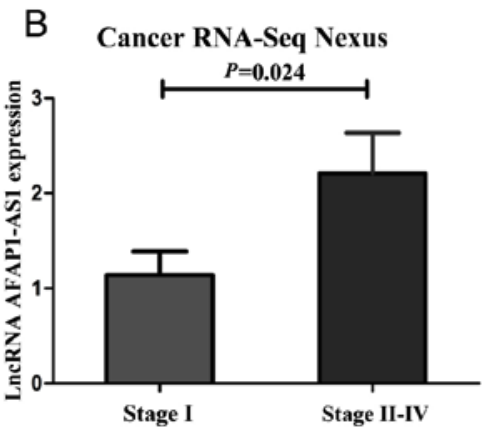

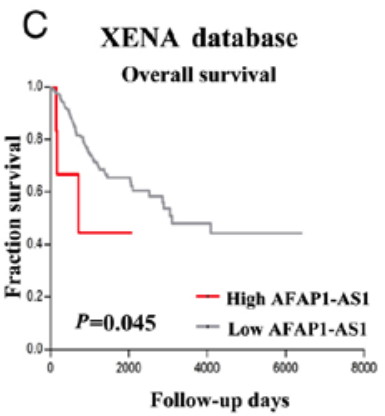

Figure 1. A high AFAP1-AS1 expression is associated with a poor prognosis of patients with cervical cancer. (A) AFAP1-AS1 expression as analyzed by TCGA cervical cancer cohort data from the Cancer RNA-Seq Nexus database, was upregulated in cervical cancer tissues (Tumor) compared to non-tumor tissues (Normal) $(\mathrm{P}<0.001)$. (B) AFAP1-AS1 expression was higher in stage II-IV lesions than in stage I lesions $(\mathrm{P}=0.024)$. (C) The cervical cancer patients with a higher AFAP1-AS1 expression have a shorter survival time. The overall survival data were collected from the TCGA datasets and analyzed using a Kaplan-Meier curve ( $\mathrm{P}=0.045)$. AFAP1-AS1, AFAP1 antisense RNA1.

A

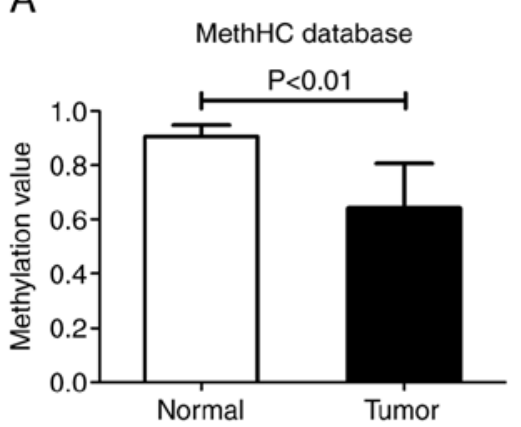

B

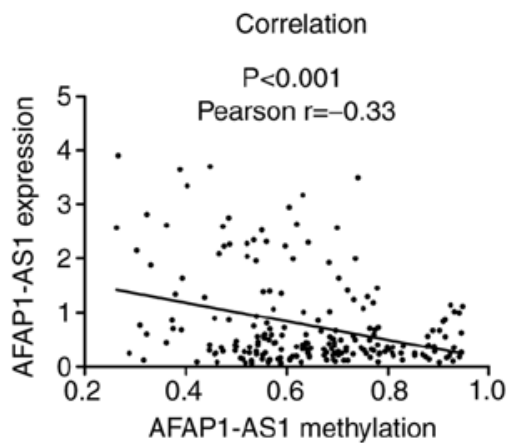

C XENA database

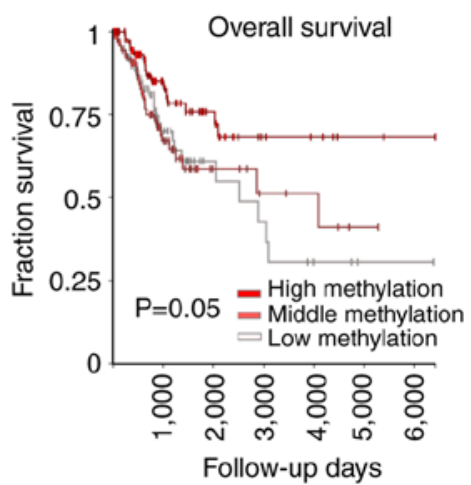

Figure 2. Hypomethylated AFAP1-AS1 is associated with a poor prognosis of patients with cervical cancer. (A) Comparisons of AFAP1-AS1 methylation level between cancer tissues (Tumor) and non-cancerous tissues (Normal) of cervical cancer involved in the TCGA based on the MethHC database ( $\mathrm{P}<0.01$ ). (B) A negative correlation between AFAP1-AS1 methylation level and AFAP1-AS1 expression in cervical cancer ( $\mathrm{r}=-0.33$, $\mathrm{P}<0.001)$. (C) Kaplan-Meier curve between groups with AFAP1-AS1 high, middle and low methylation ( $\mathrm{P}=0.05)$. This graph was conducted by XENA. AFAP1-AS1, AFAP1 antisense RNA1.
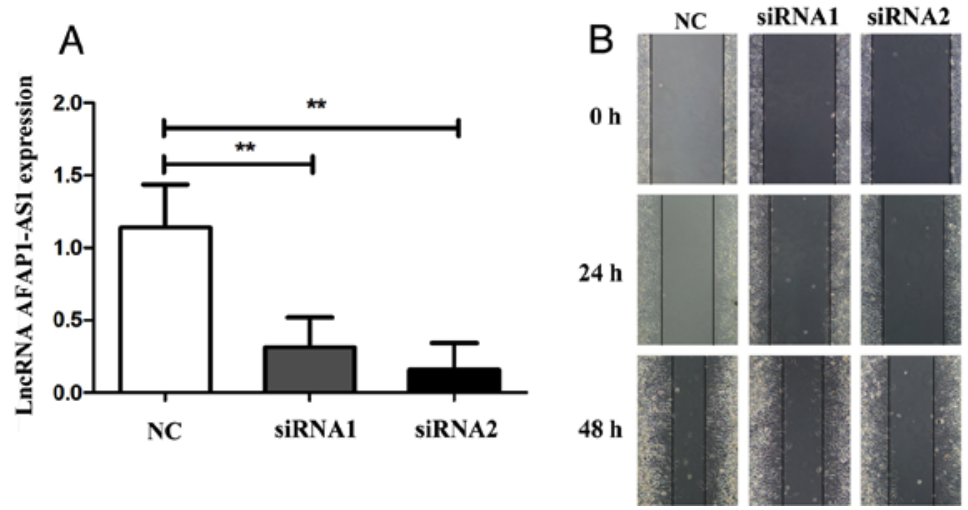

C

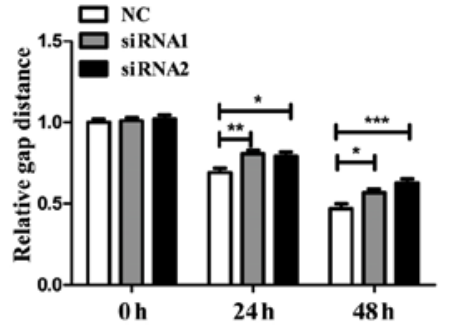

Figure 3. Cell migration is suppressed after AFAP1-AS1 knockdown. (A) Both siRNAs were effective in knocking down AFAP1-AS1 expression. siRNA1 and siRNA2 markedly suppressed AFAP1-AS1 expression compared with the scrambled control siRNA (NC) in the HeLa cells. (B) The migratory capacity of HeLa cells was suppressed after AFAP1-AS1 knockdown. The migration distance of the tumor cells were measured at 0, 24 and $48 \mathrm{~h}$. (C) The migration rate was calculated at identified time points. The AFAP1-AS1 siRNA treated cells displayed wider gap. ${ }^{*} \mathrm{P}<0.05,{ }^{* * *} \mathrm{P}<0.01$ and ${ }^{* * * *} \mathrm{P}<0.001$ vs. NC. AFAP1-AS1, AFAP1 antisense RNA1.

scratch test results revealed that the migratory capacity of the AFAP1-AS1-silenced cells was suppressed at 24 and $48 \mathrm{~h}$ following transfection with siRNA compared to the si-control group ( $24 \mathrm{~h}$, siRNA1 vs. control, $\mathrm{P}<0.01$; siRNA2 vs. control, $\mathrm{P}<0.05 ; 48 \mathrm{~h}$, siRNA1 vs. control, $\mathrm{P}<0.05 ;$ siRNA2 vs. control, $\mathrm{P}<0.001$; Fig. $3 \mathrm{~B}$ and $\mathrm{C}$ ). Moreover, as shown in Fig. 4, the number of invaded cells was also decreased in the AFAP1-AS1 siRNA-transfected cancer cells compared with 
A

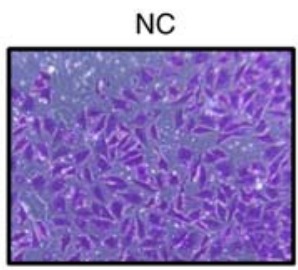

siRNA1

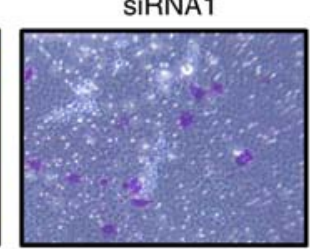

SiRNA2

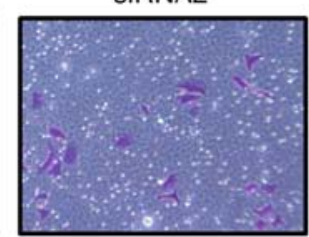

B

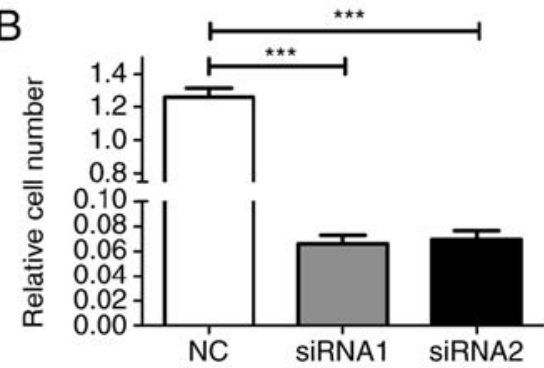

Figure 4. AFAP1-AS1 knockdown inhibits cell invasion. (A) HeLa cells were treated with AFAP1-AS1 siRNA or control siRNA, respectively. The cells were then added to the top of the matrigel invasion chambers. The results revealed that the number of invasion tumor cells was reduced after AFAP1-AS1 knockdown. (B) The representative graph of each group was presented. ${ }^{* * *} \mathrm{P}<0.001$. AFAP1-AS1, AFAP1 antisense RNA1.
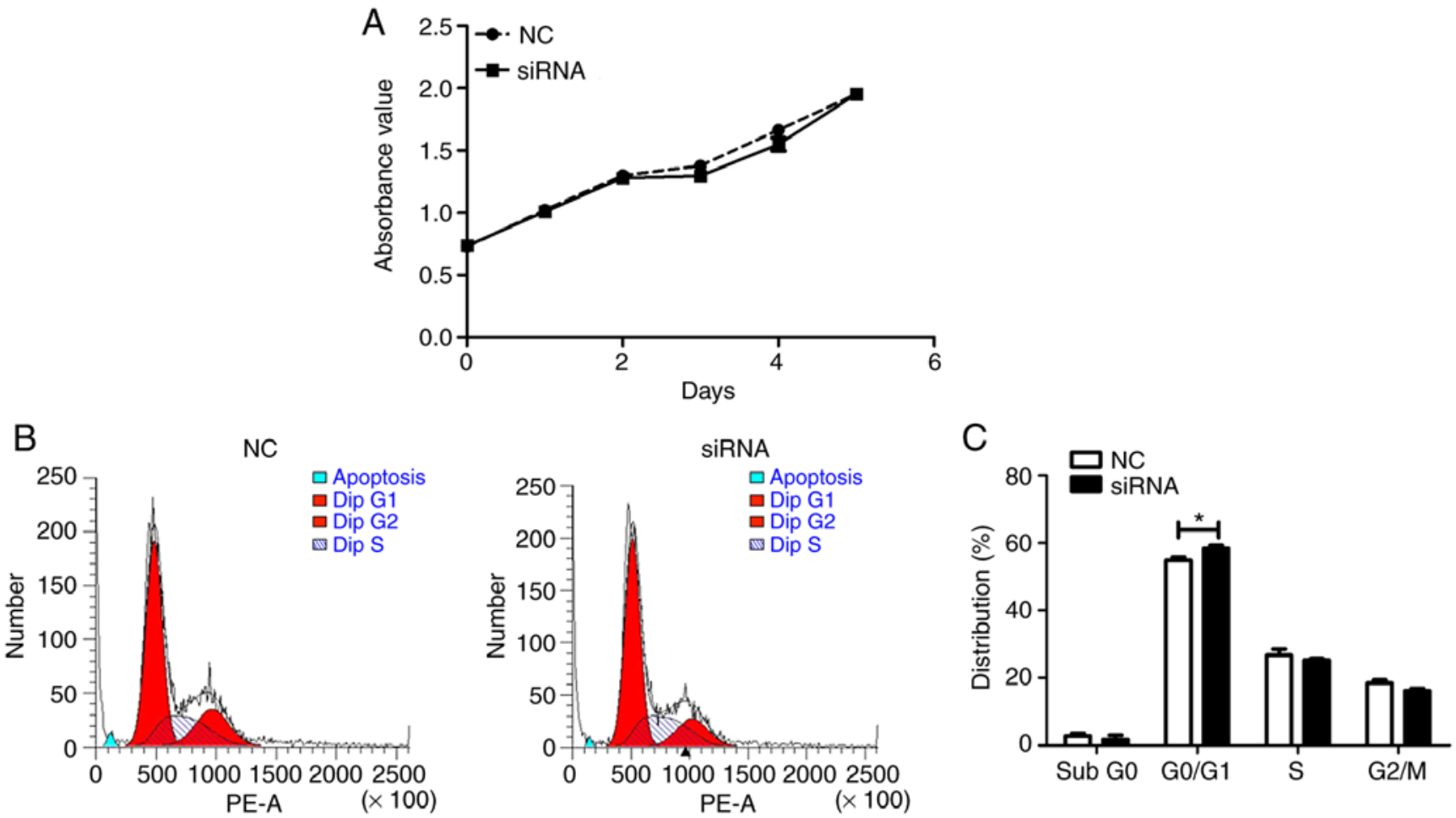

Figure 5. AFAP1-AS1 knockdown does affect cell viability and apoptosis, but slightly affects cell cycle distribution (A). The cell viability of each group at different time points was detected by MTT assay. Flow cytometric analysis was performed to (B) the analyze cell cycle and (C) apoptosis. However, no obvious difference was observed in cell apoptosis between AFAP1-AS1 knockdown group and NC group. In contrast, AFAP1-AS1 knockdown slightly, yet significantly influenced the cell cycle. "P<0.05. AFAP1-AS1, AFAP1 antisense RNA1.

the si-control group (both siRNAs, $\mathrm{P}<0.001$; Fig. 4). However, we noted that there was no significant association between the expression of AFAP1-AS1 and cell viability and cell apoptosis. By contrast, AFAP1-AS1 knockdown slightly, yet significantly increased the number of cells in the G0/G1 phase in the present study $(\mathrm{P}<0.05$; Fig. 5).

AFAP1-AS1 is involved in the regulation of the Rho/Rac signaling pathways. To investigate the potential mechanisms of AFAP1-AS1 regarding its promoting effect on the migration and invasion of cervical cancer cells, we examined the expression levels of several key molecules of the Rho/Rac signaling pathways by western blot analysis. The results suggested that the knockdown of AFAP1-AS1 increased the expression of RHOGDI, PFN1, RAB11A and RAC2, while it decreased the expression of RAB1B and LASP1 (Fig. 6).
Silencing of AFAPI-ASI affects the expression of EMT-related genes. In order to further investigate the mechanisms underlying the role of AFAP1-AS1 in cervical cancer development, we also detected changes in the protein levels of some EMT-related genes. The results revealed that following the knockdown of AFAP1-AS1, the expression of ZO-1 increased, while the expression of Vimentin and $\beta$-catenin decreased. However, E-cadherin expression was not markedly altered (Fig. 7).

\section{Discussion}

In recent years, an increasing number of studies have reported that non-coding RNAs, including IncRNAs, miRNAs and circular RNAs (circRNAs) act as tumor suppressors or promoters in tumorigenesis and pathogenesis (44-46). 


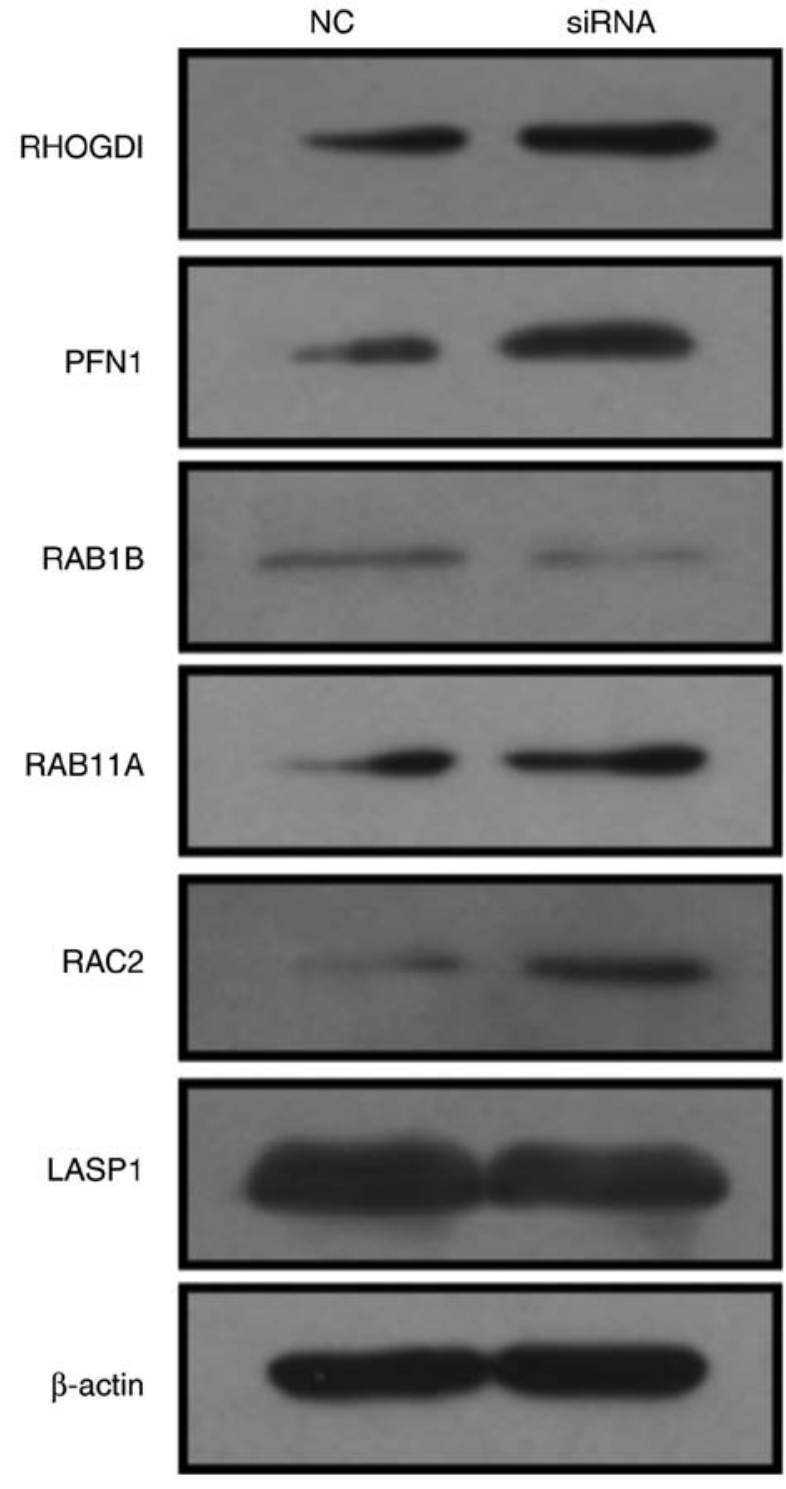

Figure 6. The expression levels of Rho/Rac GTPase family members were altered after AFAP1-AS1 knockdown. Cells were transfected with AFAP1-AS1 siRNA or control siRNA, respectively. The protein levels of Rho/Rac GTPase family members were detected by western blot analysis. The protein levels of RHOGDI, PFN1, RAB11A and RAC2 were elevated, while RAB1B and LASP1 protein levels were decreased. AFAP1-AS1, AFAP1 antisense RNA1.

Accumulating evidence indicates that the abnormal expression of lncRNAs is associated with tumorigenesis and progression (43,47-52). The TCGA database includes genome, transcriptome, epigenetic, proteomic and clinical phenotypic data of 34 tumors, which is one of the most authoritative cancer databases worldwide (53-55). Based on the TCGA database, researchers developed the MethCH, XENA and CRN databases. These provide numerous transcriptome, epigenetic and clinical phenotypic data for data mining about human malignant tumors. The mining of these data is a frequently used and convenient method for the exploration of gene or IncRNA expression and function (43).

In the present study, we combined the MethCH, XENA and $\mathrm{CRN}$ databases to investigate whether the transcription level and methylation level of lncRNA AFAP1-AS1 differ in cervical cancer. We found that AFAP1-AS1 was upregulated and

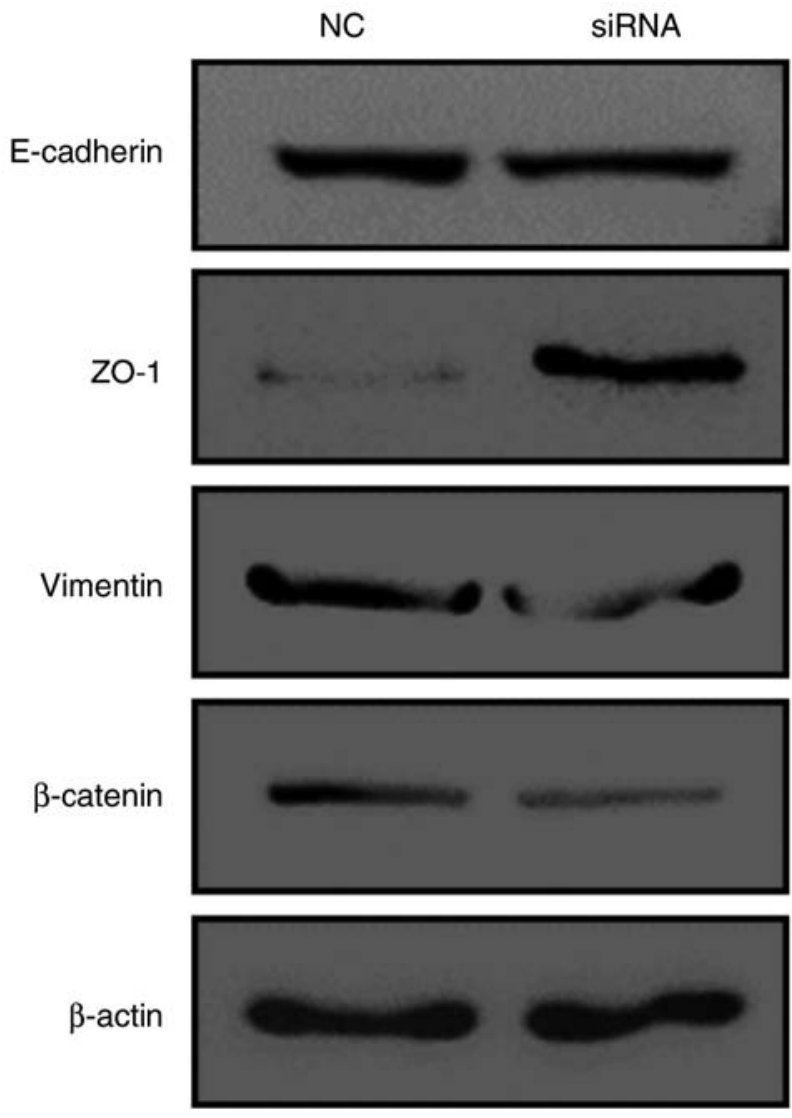

Figure 7. The expression of EMT-related genes were decreased after AFAP1-AS1 knockdown. The expression levels of vimentin and $\beta$-catenin were downregulated, while those of ZO-1 were upregulated in si-AFAP1-AS1-transfected HeLa cells compared to the si-control group. E-cadherin expression was not markedly altered. EMT, epithelial-mesenchymal transition; AFAP1-AS1, AFAP1 antisense RNA1; ZO-1, Zonula occludens-1.

hypomethylated in cervical cancer tissues compared to normal tissues, which is consistent with the findings of a previous study showing that the increased expression of AFAP1-AS1 was due to its promoter demethylation in esophageal carcinoma (30). Moreover, AFAP1-AS1 upregulation and hypomethylation were associated with a shorter survival time of patients with cervical cancer. In addition, some studies have revealed that lncRNA AFAP1-AS1 is enriched in several tumors and functions as an oncogene $(19,20,30,56,57)$. Thus, we hypothesized that AFAP1-AS1 may also function as an oncogene in cervical cancer. Subsequent functional experiments confirmed that cell migration and invasion were significantly suppressed following the knockdown of AFAP1-AS1 in cervical cancer cells, while no effect on cell proliferation or cell apoptosis in vitro were observed. However, AFAP1-AS1 knockdown slightly affected cell cycle distribution.

The Rho/Rac GTPase family is an important member of the Ras superfamily. It regulates cytoskeletal remodeling by affecting the polymerization and stability of microtubules and microfilaments. The Rho/Rac GTPase family has been reported to regulate cell adhesion and motility (58-60). Several studies have found that Rho/Rac GTPase family members are involved in the migration and invasion of various tumors, including colorectal cancer and ovarian cancer $(61,62)$. Moreover, two recent studies indicated that IncRNA AFAP1-AS1 was involved 
in the regulation of Rho/Rac GTPase family members $(19,56)$. In the present study, we determined that the RHOGDI, PFN1, RAB11A and RAC2 protein levels were elevated, while the RAB1B and LASP1 protein levels were suppressed after the silencing of AFAP1-AS1 in HeLa cells. These results were consistent with those of previous studies on lung cancer and nasopharyngeal carcinoma $(19,20)$. However, the change in RAC2 protein levels was contrary to the findings of a previous study on liver cancer (56). This suggests that AFAP1-AS1 may modulate the Rho/Rac pathway through different mechanisms in different types of tumors.

EMT is a vital malignant phenotype in tumor cell migration and invasion which promotes tumor development $(48,63)$. A number of studies have indicated that lncRNAs play important roles in the EMT process in tumors (64-69). In the present study, to identify whether AFAP1-AS1 regulates the EMT process in cervical cancer as well, the protein levels of some EMT-related genes were measured by western blot analysis. The data suggested that AFAP1-AS1 knockdown downregulated the EMT-related genes vimentin and $\beta$-catenin, while it upregulated ZO-1 in cervical cancer cells. Our data indicated that AFAP1-AS1 may function as a tumor promoter by affecting the EMT process in HeLa cells. $\beta$-catenin is an important member of the Wnt/ $\beta$-catenin signaling pathway. We demonstrated that AFAP1-AS1 knockdown decreased the protein levels of $\beta$-catenin. Nevertheless, it is not clear whether the Wnt/ $\beta$-catenin signaling pathway suggests a downstream of AFAP1-AS1 in cervical cancer. This question warrants further investigation. In addition, only one cell line was used in the present study to explore the function and mechanism of AFAP1-AS1 in cervical cancer. The lack of multiple cell lines in the present study was a limitation, and that the results should be confirmed in further experimental models.

Taken together, the findings of the present study revealed that AFAP1-AS1 was significantly hypomethylated and upregulated in cervical cancer. And hypomethylation and upregulation of AFAP1-AS1 are both associated with a poor outcome of patients with cervical cancer. We further confirmed that AFAP1-AS1 knockdown suppressed the migration and invasion of HeLa cells. The inhibition of AFAP1-AS1 negatively regulated the Rho/Rac pathway and EMT-related gene protein products. The identified lncRNA AFAP1-AS1 may be a novel target for cervical cancer therapy.

\section{Acknowledgements}

The authors would like to thank Dr Wei Wang (Department of Pathology, Affiliated Hospital of Jining Medical University, Jining, Shandong, China) for providing excellent technical assistance.

\section{Funding}

The present study was supported by grants from The National Natural Science Foundation of China (nos. 81572787, 81672683, 81672993, 81672688, 81702907, 81772901,81772928,81803025 and 81872278), the Overseas Expertise Introduction Project for Discipline Innovation (111 Project, no. 111-2-12), the Natural Science Foundation of Hunan Province (nos. 2016JC2035, 2017SK2105, 2018JJ3704, 2018JJ3815, 2018SK21210 and
2018SK21211), the Foundation from the Changsha Science and Technology Board (kq1706045) and Chinese Anti-Cancer Association, the Fundamental Research Funds for the Central Universities of Central South University (no. 1053320171023) and the Special Fund of Clinical Medicine of Chinese Medical Association (no. 17020280697).

\section{Availability of data and materials}

All data generated or analyzed during the present study are included in this published article or are available from the corresponding author on reasonable request.

\section{Authors' contributions}

$\mathrm{HB}$ and ZG mainly performed the experiments, analyzed the data and wrote the paper. ZL, LS, CG, XL, QL, WZ, KC and MZ helped with the experiments and analyzed the data. BX, XL, QL and LF helped with the data acquisition. BX, XL, WX, LF, ZZ, SZ and FX helped with the paper writing. GL, WX, ZZ, SZ and FX carried out the experiment design and manuscript drafting. All authors read and approved the manuscript and agree to be accountable for all aspects of the research in ensuring that the accuracy or integrity of any part of the work are appropriately investigated and resolved.

\section{Ethics approval and consent to participate}

All patient data analyzed in the present study were downloaded from online databases. Thus, no ethics approval was required.

\section{Patient consent for publication}

Not applicable.

\section{Competing interests}

The authors declare that they have no competing interests.

\section{References}

1. Bray F, Ferlay J, Soerjomataram I, Siegel RL, Torre LA and Jemal A: Global cancer statistics 2018: GLOBOCAN estimates of incidence and mortality worldwide for 36 cancers in 185 countries. CA Cancer J Clin 68: 394-424, 2018.

2. Torre LA, Islami F, Siegel RL, Ward EM and Jemal A: Global Cancer in Women: Burden and Trends. Cancer Epidemiol Biomarkers Prev 26: 444-457, 2017.

3. Wei F, Wu Y, Tang L, Xiong F, Guo C, Li X, Zhou M, Xiang B, Li X, Li G, et al: Trend analysis of cancer incidence and mortality in China. Sci China Life Sci 60: 1271-1275, 2017.

4. Deng X, Xiong F, Li X, Xiang B, Li Z, Wu X, Guo C, Li X, Li Y, $\mathrm{Li} \mathrm{G}$, et al: Application of atomic force microscopy in cancer research. J Nanobiotechnology 16: 102, 2018.

5. Cancer Genome Atlas Research Network; Albert Einstein College of Medicine; Analytical Biological Services; Barretos C ancer Hospital; Baylor College of Medicine; Beckman Research Institute of City of Hope; Buck Institute for Research on Aging; Canada's Michael Smith Genome Sciences Centre; Harvard Medical School; Helen F. Graham Cancer Center \& Research Institute at Christiana Care Health Services, et al: Integrated genomic and molecular characterization of cervical cancer. Nature 543: 378-384, 2017.

6. Xie G, Wang Z, Chen Y, Zhang S, Feng L, Meng F and Yu Z: Dual blocking of PI3K and mTOR signaling by NVP-BEZ235 inhibits proliferation in cervical carcinoma cells and enhances therapeutic response. Cancer Lett 388: 12-20, 2017. 
7. Liao S, Xiao S, Chen H, Zhang M, Chen Z, Long Y, Gao L, Zhu G, He J, Peng S, et al: CD38 enhances the proliferation and inhibits the apoptosis of cervical cancer cells by affecting the mitochondria functions. Mol Carcinog 56: 2245-2257, 2017.

8. Gong Z, Zhang S, Zhang W, Huang H, Li Q, Deng H, Ma J, Zhou M, Xiang J, Wu M, et al: Long non-coding RNAs in cancer. Sci China Life Sci 55: 1120-1124, 2012.

9. Tang Y, Wang J, Lian Y, Fan C, Zhang P, Wu Y, Li X, Xiong F, Li X, Li G, et al: Linking long non-coding RNAs and SWI/SNF complexes to chromatin remodeling in cancer. Mol Cancer 16: 42, 2017.

10. Yang L, Tang Y, Xiong F, He Y, Wei F, Zhang S, Guo C, Xiang B, Zhou M, Xie N, et al: LncRNAs regulate cancer metastasis via binding to functional proteins. Oncotarget 9: 1426-1443, 2018.

11. Wang JP, Tang YY, Fan CM, He Y, Wei F, Zhang S, Guo C, Xiang $\mathrm{B}$, Zhou M, Xie N, et al: The role of exosomal non-coding RNAs in cancer metastasis. Oncotarget 9: 12487-12502, 2018.

12. Fan C, Tang Y, Wang J, Xiong F, Guo C, Wang Y, Zhang S, Gong Z, Wei F, Yang L, et al: Role of long non-coding RNAs in glucose metabolism in cancer. Mol Cancer 16: 130, 2017.

13. Gong Z, Yang Q, Zeng Z, Zhang W, Li X, Zu X, Deng H, Chen P, Liao Q, Xiang B, et al: An integrative transcriptomic analysis reveals p53 regulated miRNA, mRNA, and lncRNA networks in nasopharyngeal carcinoma. Tumour Biol 37: 3683-3695, 2016.

14. Fan C, Wang J, Tang Y, Wang Y, Xiong F, Zhang S, Li X, Xiang B, Wu X, Guo C, et al: Long non-coding RNA LOC284454 promotes migration and invasion of nasopharyngeal carcinoma via modulating the Rho/Rac signaling pathway. Carcinogenesis: Oct 30, 2018 (Epub ahead of print). doi: 10.1093/carcin/bgy143.

15. He Y, Jing Y, Wei F, Tang Y, Yang L, Luo J, Yang P, Ni Q, Pang J, Liao Q, et al: Long non-coding RNA PVT1 predicts poor prognosis and induces radioresistance by regulating DNA repair and cell apoptosis in nasopharyngeal carcinoma. Cell Death Dis 9: $235,2018$.

16. Yu J, Liu Y, Guo C, Zhang S, Gong Z, Tang Y, Yang L, $\mathrm{He} \mathrm{Y}$, Lian Y, Li X, et al: Upregulated long non-coding RNA LINC00152 expression is associated with progression and poor prognosis of tongue squamous cell carcinoma. J Cancer 8: 523-530, 2017

17. Yu J, Liu Y, Gong Z, Zhang S, Guo C, Li X, Tang Y, Yang L, He Y, Wei F, et al: Overexpression long non-coding RNA LINC00673 is associated with poor prognosis and promotes invasion and metastasis in tongue squamous cell carcinoma. Oncotarget 8 : 16621-16632, 2017

18. Wang Y, Xue D, Li Y, Pan X, Zhang X, Kuang B, Zhou M, Li X, Xiong $\mathrm{W}, \mathrm{Li} \mathrm{G}$, et al: The long noncoding RNA MALAT-1 is a novel biomarker in various cancers: A meta-analysis based on the GEO database and literature. J Cancer 7: 991-1001, 2016

19. Bo H, Gong Z, Zhang W, Li X, Zeng Y, Liao Q, Chen P, Shi L, Lian Y, Jing Y, et al: Upregulated long non-coding RNA AFAP1-AS1 expression is associated with progression and poor prognosis of nasopharyngeal carcinoma. Oncotarget 6 : 20404-20418, 2015

20. Zeng Z, Bo H, Gong Z, Lian Y, Li X, Li X, Zhang W, Deng H, Zhou M, Peng S, et al: AFAP1-AS1, a long noncoding RNA upregulated in lung cancer and promotes invasion and metastasis. Tumour Biol 37: 729-737, 2016.

21. Wang Y, Mo Y, Yang X, Zhou R, Wu Z, He Y, Yang X, Zhong Y, Du Y, Zhou $\mathrm{H}$, et al: Long non-coding RNA AFAP1-AS1 is a novel biomarker in various cancers: A systematic review and meta-analysis based on the literature and GEO datasets. Oncotarget 8: 102346-102360, 2017.

22. Tang Y, He Y, Shi L, Yang L, Wang J, Lian Y, Fan C, Zhang P Guo C, Zhang S, et al: Co-expression of AFAP1-AS1 and PD-1 predicts poor prognosis in nasopharyngeal carcinoma. Oncotarget 8: 39001-39011, 2017.

23. Lian Y, Xiong F, Yang L, Bo H, Gong Z, Wang Y, Wei F, Tang Y, Li X, Liao Q, et al: Long noncoding RNA AFAP1-AS1 acts as a competing endogenous RNA of miR-423-5p to facilitate nasopharyngeal carcinoma metastasis through regulating the Rho/Rac pathway. J Exp Clin Cancer Res 37: 253, 2018.

24. Bo H, Fan L, Li J, Liu Z, Zhang S, Shi L, Guo C, Li X, Liao Q, Zhang W, et al: High expression of IncRNA AFAP1-AS1 promotes the progression of colon cancer and predicts poor prognosis. J Cancer 9: 4677-4683, 2018.

25. Guo JQ, Li SJ and Guo GX: Long noncoding RNA AFAP1-AS1 promotes cell proliferation and apoptosis of gastric cancer cells via PTEN/p-AKT pathway. Dig Dis Sci 62: 2004-2010, 2017.

26. Han X, Wang L, Ning Y, Li S and Wang Z: Long non-coding RNA AFAP1-AS1 facilitates tumor growth and promotes metastasis in colorectal cancer. Biol Res 49: 36, 2016.
27. Deng J, Liang Y, Liu C, He S and Wang S: The up-regulation of long non-coding RNA AFAP1-AS1 is associated with the poor prognosis of NSCLC patients. Biomed Pharmacother 75: 8-11, 2015.

28. Li JR, Sun CH, Li W, Chao RF, Huang CC, Zhou XJ and Liu CC Cancer RNA-Seq Nexus: A database of phenotype-specific transcriptome profiling in cancer cells. Nucleic Acids Res 44: D944-951, 2016 .

29. Huang WY, Hsu SD, Huang HY, Sun YM, Chou CH, Weng SL and Huang HD: MethHC: A database of DNA methylation and gene expression in human cancer. Nucleic Acids Res 43: D856-D861, 2015.

30. Wu W, Bhagat TD, Yang X, Song JH, Cheng Y, Agarwal R, Abraham JM, Ibrahim S, Bartenstein M, Hussain Z, et al: Hypomethylation of noncoding DNA regions and overexpression of the long noncoding RNA, AFAPI-ASI, in Barrett's esophagus and esophageal adenocarcinoma. Gastroenterology 144: 956-966 e954, 2013.

31. Wei F, Tang L, He Y, Wu Y, Shi L, Xiong F, Gong Z, Guo C, Li X, Liao Q, et al: BPIFB1 (LPLUNC1) inhibits radioresistance in nasopharyngeal carcinoma by inhibiting VTN expression. Cell Death Dis 9: 432, 2018.

32. Song Y, Li X, Zeng Z, Li Q, Gong Z, Liao Q, Li X, Chen P, Xiang B, Zhang W, et al: Epstein-Barr virus encoded miR-BART11 promotes inflammation-induced carcinogenesis by targeting FOXP1. Oncotarget 7: 36783-36799, 2016.

33. Zeng Z, Huang H, Huang L, Sun M, Yan Q, Song Y, Wei F, Bo H, Gong Z, Zeng Y, et al: Regulation network and expression profiles of Epstein-Barr virus-encoded microRNAs and their potential target host genes in nasopharyngeal carcinomas. Sci China Life Sci 57: 315-326, 2014.

34. Yi M, Wang W, Chen S, Peng Y, Li J, Cai J, Zhou Y, Peng Q, Ban Y, Zeng Z, et al: Dual-functionality of RASSF1A overexpression in A375 cells is mediated by activation of IL-6/STAT3 regulatory loop. Mol Biol Rep 45: 1277-1287, 2018.

35. Zou G, Ren B, Liu Y, Fu Y, Chen P, Li X, Luo S, He J, Gao G, Zeng $Z$, et al: Inhibin B suppresses anoikis resistance and migration through the transforming growth factor- $\beta$ signaling pathway in nasopharyngeal carcinoma. Cancer Sci 109: 3416-3427, 2018.

36. He R, Liu P, Xie X, Zhou Y, Liao Q, Xiong W, Li X, Li G, Zeng Z and Tang H: circGFRA1 and GFRA1 act as ceRNAs in triple negative breast cancer by regulating miR-34a. J Exp Clin Cancer Res 36: 145, 2017.

37. Liao Q, Zeng Z, Guo X, Li X, Wei F, Zhang W, Li X, Chen P, Liang F, Xiang B, et al: LPLUNC1 suppresses IL-6-induced nasopharyngeal carcinoma cell proliferation via inhibiting the Stat3 activation. Oncogene 33: 2098-2109, 2014.

38. Yang Y, Liao Q, Wei F, Li X, Zhang W, Fan S, Shi L, Li X, Gong Z, Ma J, et al: LPLUNC1 inhibits nasopharyngeal carcinoma cell growth via down-regulation of the MAP kinase and cyclin D1/E2F pathways. PLoS One 8: e62869, 2013.

39. Liang F, Li Q, Li X, Li Z, Gong Z, Deng H, Xiang B, Zhou M, $\mathrm{Li} \mathrm{X}, \mathrm{Li} \mathrm{G}$, et al: TSC22D2 interacts with PKM2 and inhibits cell growth in colorectal cancer. Int J Oncol 49: 1046-1056, 2016.

40. Li Q, Chen P, Zeng Z, Liang F, Song Y, Xiong F, Li X, Gong Z, Zhou M, Xiang B, et al: Yeast two-hybrid screening identified WDR77 as a novel interacting partner of TSC22D2. Tumour Biol 37: 12503-12512, 2016.

41. Livak KJ and Schmittgen TD: Analysis of relative gene expression data using real-time quantitative PCR and the $2^{-\Delta \Delta C_{\mathrm{T}}}$ method. Methods 25: 402-408, 2001.

42. Zhou Y, Zhang X and Klibanski A: MEG3 noncoding RNA: A tumor suppressor. J Mol Endocrinol 48: R45-R53, 2012.

43. Wang Z, Yang B, Zhang M, Guo W, Wu Z, Wang Y, Jia L, Li S; Cancer Genome Atlas Research Network, Xie W and Yang D: lncRNA epigenetic landscape analysis identifies EPIC1 as an oncogenic lncRNA that interacts with MYC and promotes cell-cycle progression in cancer. Cancer Cell 33: 706-720.e9, 2018.

44. Wang Y, Mo Y, Gong Z, Yang X, Yang M, Zhang S, Xiong F, Xiang B, Zhou M, Liao Q, et al: Circular RNAs in human cancer. Mol Cancer 16: 25, 2017.

45. Zhong Y, Du Y, Yang X, Mo Y, Fan C, Xiong F, Ren D, Ye X, Li C, Wang Y, et al: Circular RNAs function as ceRNAs to regulate and control human cancer progression. Mol Cancer 17: 79, 2018.

46. Zhou R, Wu Y, Wang W, Su W, Liu Y, Wang Y, Fan C, Li X, Li G, Li Y, et al: Circular RNAs (circRNAs) in cancer. Cancer Lett 425: 134-142, 2018. 
47. Yuan JH, Yang F, Wang F, Ma JZ, Guo YJ, Tao QF, Liu F, Pan W, Wang TT, Zhou CC, et al: A long noncoding RNA activated by TGF- $\beta$ promotes the invasion-metastasis cascade in hepatocellular carcinoma. Cancer Cell 25: 666-681, 2014.

48. He B, Li W, Wu Y, Wei F, Gong Z, Bo H, Wang Y, Li X, Xiang B, Guo C, et al: Epstein-Barr virus-encoded miR-BART6-3p inhibits cancer cell metastasis and invasion by targeting long non-coding RNA LOC553103. Cell Death Dis 7: e2353, 2016.

49. Gong Z, Zhang S, Zeng Z, Wu H, Yang Q, Xiong F, Shi L, Yang J, Zhang W, Zhou Y, et al: LOC401317, a p53-regulated long non-coding RNA, inhibits cell proliferation and induces apoptosis in the nasopharyngeal carcinoma cell line HNE2. PLoS One 9: e110674, 2014.

50. Zeng Z, Fan S, Zhang X, Li S, Zhou M, Xiong W, Tan M, Zhang W and Li G: Epstein-Barr virus-encoded small RNA 1 (EBER-1) could predict good prognosis in nasopharyngeal carcinoma. Clin Transl Oncol 18: 206-211, 2016.

51. Xu K, Xiong W, Zhou M, Wang H, Yang J, Li X, Chen P, Liao Q, Deng $\mathrm{H}, \mathrm{Li} \mathrm{X}$, et al: Integrating ChIP-sequencing and digital gene expression profiling to identify BRD7 downstream genes and construct their regulating network. Mol Cell Biochem 411: 57-71, 2016.

52. Zhang W, Huang C, Gong Z, Zhao Y, Tang K, Li X, Fan S, Shi L, Li X, Zhang P, et al: Expression of LINC00312, a long intergenic non-coding RNA, is negatively correlated with tumor size but positively correlated with lymph node metastasis in nasopharyngeal carcinoma. J Mol Histol 44: 545-554, 2013.

53. Tu C,Zeng Z, Qi P,Li X, Guo C, Xiong F, Xiang B,Zhou M,Liao Q, $\mathrm{Yu}$ J,et al: Identification of genomic alterations in nasopharyngeal carcinoma and nasopharyngeal carcinoma-derived Epstein-Barr virus by whole genome sequencing. Carcinogenesis 39: 1517-1528, 2018.

54. Tu C, Zeng Z, Qi P, Li X, Yu Z, Guo C, Xiong F, Xiang B, Zhou M, Gong Z, et al: Genome-wide analysis of 18 Epstein-Barr viruses isolated from primary nasopharyngeal carcinoma biopsy specimens. J Virol 91: pii: e00301-17, 2017.

55. Xiao K, Yu Z, Li X, Li X, Tang K, Tu C, Qi P, Liao Q, Chen P, Zeng Z, et al: Genome-wide analysis of Epstein-Barr virus (EBV) integration and strain in C666-1 and Raji cells. J Cancer 7: 214-224, 2016

56. Zhang JY, Weng MZ, Song FB, Xu YG, Liu Q, Wu JY, Qin J, Jin T and Xu JM: Long noncoding RNA AFAP1-AS1 indicates a poor prognosis of hepatocellular carcinoma and promotes cell proliferation and invasion via upregulation of the RhoA/Rac2 signaling. Int J Oncol 48: 1590-1598, 2016.

57. Lu X, Zhou C, Li R, Deng Y, Zhao L and Zhai W: Long noncoding RNA AFAP1-AS1 promoted tumor growth and invasion in cholangiocarcinoma. Cell Physiol Biochem 42: 222-230, 2017.
58. Tang $\mathrm{Y}$, He Y, Zhang $\mathrm{P}$, Wang J, Fan C, Yang L, Xiong F, Zhang S, Gong Z, Nie S, et al: LncRNAs regulate the cytoskeleton and related Rho/ROCK signaling in cancer metastasis. Mol Cancer 17: 77, 2018

59. Wang M, Zhao J, Zhang L, Wei F, Lian Y, Wu Y, Gong Z, Zhang S, Zhou J, Cao K, et al: Role of tumor microenvironment in tumorigenesis. J Cancer 8: 761-773, 2017.

60. Yi M, Cai J, Li J, Chen S, Zeng Z, Peng Q, Ban Y, Zhou Y, $\mathrm{Li} \mathrm{X}$, Xiong $\mathrm{W}$, et al: Rediscovery of NF- $\mathrm{KB}$ signaling in nasopharyngeal carcinoma: How genetic defects of NF- $\kappa \mathrm{B}$ pathway interplay with EBV in driving oncogenesis? J Cell Physiol 233 5537-5549, 2018.

61. Gou WF, Zhao Y, Lu H, Yang XF, Xiu YL, Zhao S, Liu JM, Zhu ZT, Sun HZ, Liu YP, et al: The role of RhoC in epithelial-to-mesenchymal transition of ovarian carcinoma cells. BMC Cancer 14: 477, 2014.

62. Makrodouli E, Oikonomou E, Koc M, Andera L, Sasazuki T, Shirasawa $\mathrm{S}$ and Pintzas A: BRAF and $R A S$ oncogenes regulate Rho GTPase pathways to mediate migration and invasion properties in human colon cancer cells: A comparative study. Mol Cancer 10: 118, 2011.

63. Zhou Y, Liao Q, Li X, Wang H, Wei F, Chen J, Yang J, Zeng Z, Guo X, Chen P, et al: HYOU1, regulated by LPLUNC1, is up-regulated in nasopharyngeal carcinoma and associated with poor prognosis. J Cancer 7: 367-376, 2016.

64. Wei F, Wu Y, Tang L, He Y, Shi L, Xiong F, Gong Z, Guo C, Li X, Liao Q, et al: BPIFB1 (LPLUNC1) inhibits migration and invasion of nasopharyngeal carcinoma by interacting with VTN and VIM. Br J Cancer 118: 233-247, 2018.

65. Yang L, Tang Y, He Y, Wang Y, Lian Y, Xiong F, Shi L, Zhang S, Gong Z, Zhou Y, et al: High expression of LINC01420 indicates an unfavorable prognosis and modulates cell migration and invasion in nasopharyngeal carcinoma. J Cancer 8: 97-103, 2017.

66. Fan C, Tang Y, Wang J, Xiong F, Guo C, Wang Y, Xiang B, Zhou M, Li X, Wu X, et al: The emerging role of Epstein-Barr virus encoded microRNAs in nasopharyngeal carcinoma. J Cancer 9: 2852-2864, 2018.

67. Zhang Y, Xia M, Jin K, Wang S, Wei H, Fan C, Wu Y, Li X, $\mathrm{Li}$ X, Li G, et al: Function of the c-Met receptor tyrosine kinase in carcinogenesis and associated therapeutic opportunities. Mol Cancer 17: 45, 2018.

68. Tang L, Wei F, Wu Y, He Y, Shi L, Xiong F, Gong Z, Guo C, Li X, Deng H, et al: Role of metabolism in cancer cell radioresistance and radiosensitization methods. J Exp Clin Cancer Res 37: 87, 2018.

69. Wang YA, Li XL, Mo YZ, Fan CM, Tang L, Xiong F, Guo C, Xiang B, Zhou M, Ma J, et al: Effects of tumor metabolic microenvironment on regulatory T cells. Mol Cancer 17: 168, 2018 